\title{
Screening and identification of hub genes in bladder cancer by bioinformatics analysis and KIF11 is a potential prognostic biomarker
}

\author{
XIAO-CONG MO ${ }^{1,2^{*}}$, ZI-TONG ZHANG ${ }^{1,3^{*}}$, MENG-JIA SONG ${ }^{1,2}$, ZI-QI ZHOU ${ }^{1,2}$, \\ JIAN-XIONG ZENG ${ }^{1,2}$, YU-FEI DU ${ }^{1,2}$, FENG-ZE SUN ${ }^{1,2}$, JIE-YING YANG ${ }^{1,2}$, JUN-YI HE ${ }^{1,2}$, \\ YUE HUANG $^{1,2}$, JIAN-CHUAN XIA ${ }^{1,2}$ and DE-SHENG WENG ${ }^{1,2}$

\footnotetext{
${ }^{1}$ State Key Laboratory of Oncology in South China, Collaborative Innovation Centre for Cancer Medicine;

${ }^{2}$ Department of Biotherapy, Sun Yat-Sen University Cancer Center; ${ }^{3}$ Department of Radiation Oncology, Sun Yat-Sen University Cancer Center, Guangzhou, Guangdong 510060, P.R. China
}

Received July 31, 2020; Accepted December 18, 2020

DOI: 10.3892/ol.2021.12466

\begin{abstract}
Bladder cancer (BC) is the ninth most common lethal malignancy worldwide. Great efforts have been devoted to clarify the pathogenesis of $\mathrm{BC}$, but the underlying molecular mechanisms remain unclear. To screen for the genes associated with the progression and carcinogenesis of $\mathrm{BC}$, three datasets were obtained from the Gene Expression Omnibus. A total of 37 tumor and 16 non-cancerous samples were analyzed to identify differentially expressed genes (DEGs). Subsequently, 141 genes were identified, including 55 upregulated and 86 downregulated genes. The protein-protein interaction network was established using the Search Tool for Retrieval of Interacting Genes database. Hub gene identification and module analysis were performed using Cytoscape software Hierarchical clustering of hub genes was conducted using the University of California,Santa Cruz Cancer Genomics Browser. Among the hub genes, kinesin family member 11 (KIF11) was identified as one of the most significant prognostic biomarkers among all the candidates. The Kaplan Meier Plotter database was used for survival analysis of KIF11. The expression profile of KIF11 was analyzed using the ONCOMINE database. The expression levels of KIF11 in BC samples and bladder cells were measured using reverse transcription-quantitative $\mathrm{pCR}$,
\end{abstract}

Correspondence to: Professor De-Sheng Weng or Professor Jian-Chuan Xia, Department of Biotherapy, Sun Yat-Sen University Cancer Center, 651 Dongfeng East Road, Guangzhou, Guangdong 510060, P.R. China

E-mail:wengdsh@sysucc.org.cn

E-mail: xiajch@sysucc.org.cn

*Contributed equally

Key words: bladder cancer, bioinformatics analysis, kinesin family member 11, biomarker immunohistochemistry and western blotting. In summary, KIF11 was significantly upregulated in BC and might act as a potential prognostic biomarker. The present identification of DEGs and hub genes in BC may provide novel insight for investigating the molecular mechanisms of $\mathrm{BC}$.

\section{Introduction}

Bladder cancer (BC) is the ninth most common malignancy worldwide with substantial morbidity and mortality. In the United States in 2019, there were an estimated 80,470 new cases and 17,670 deaths due to bladder cancer (1). The incidence rate of $\mathrm{BC}$ is increasing rapidly (2). Therefore, developing precise diagnostic strategies and treatments are necessary. Though numerous previous studies have revealed several insights into the pathogenesis of $\mathrm{BC}$, the underlying molecular mechanisms are largely unknown (3-5). To increase the diagnostic and therapeutic accuracy of $\mathrm{BC}$, it is necessary to explore the underlying molecular mechanisms.

In the present study, three original microarray datasets [GSE37817 (6), GSE42089 (7) and GSE52519(8)] were obtained from the Gene Expression Omnibus (GEO) database to identify differentially expressed genes (DEGs) in bladder tumors and non-cancerous samples. Afterwards, Gene Ontology (GO) and Kyoto Encyclopedia of Genes and Genomes (KEGG) analyses and PPI were conducted to explore the molecular mechanisms of tumorigenesis and progression. These hub genes were identified and analyzed using the cBioPortal online platform. In addition, upregulation of KIF11 was verified in BC samples and bladder cells. The expression levels of KIF11 were measured using reverse transcription-quantitative PCR, immunohistochemistry analysis and western blotting.

\section{Materials and methods}

Microarray data. The GEO database (9) is a publicly available functional genomics database, including microarrays, chips and high-throughput gene expression data. Three datasets downloaded from GEO were used in the present 
Table I. Detailed information for datasets downloaded from the GEO database.

\begin{tabular}{llccl}
\hline $\begin{array}{l}\text { Series } \\
\text { accession }\end{array}$ & \multicolumn{1}{c}{ Country } & Year & $\begin{array}{c}\text { Sample size } \\
\text { case/control }\end{array}$ & Platform \\
\hline GSE37817 & South Korea & 2012 & $18 / 5$ & GPL6102 Illumina human-6 v2.0 expression beadchip \\
GSE42089 & United States of America & 2012 & $10 / 8$ & GPL9828 Affymetrix Human Genome U133 Plus 2.0 Array \\
GSE52519 & Russia & 2013 & $9 / 3$ & GPL6884 Illumina HumanWG-6 v3.0 expression beadchip \\
\hline
\end{tabular}

GEO, Gene Expression Omnibus.

study, including GSE37817, GSE42089 and GSE52519. The GSE37817 dataset contained 18 BC tissue samples and five non-cancerous samples. GSE42089 contained 10 tissues from BC and eight tissue samples from normal bladder. GSE52519 contained nine $\mathrm{BC}$ cancerous tissue samples and three normal bladder tissue samples. Detailed information of the three gene expression datasets are presented in Table I.

DEGs analysis. GEO2R tool (http://www.ncbi.nlm.nih. gov/geo/geo2r) was applied to screen the DEGs in BC and non-cancerous samples, which compared different datasets to identify DEGs in a GEO series. The adjusted P-value (adj. P) and Benjamini and HBChberg false discovery rate were used to identify statistically significant DEGs and rule out false-positives. An adj. P<0.01 and a llog FC (fold-change) $\mid \geq 1$ were considered statistically significant. Genes with $>1$ probe sets or probe sets without corresponding gene symbols were averaged or removed, respectively. The overlapping DEGs among the three datasets were compared using Venn analysis.

Protein-protein interaction (PPI) network construction and module analyses. The Search Tool for the Retrieval of Interacting Genes (STRING) (version 11.0) database was used to predict and analyze the function of the PPIs using networks (10). The cut-off value of the combined score was set at $>0.4$. Subsequently, the PPI networks were drawn using Cytoscape software, which was used to visualize intermolecular interaction networks (11). The Molecular Complex Detection (MCODE) plug-in of Cytoscape, designed for clustering networks to find connected regions, was used to screen significant modules from PPI networks (12). The selection criteria for significant modules were as follows: MCODE Scores $>5$, degree cut-off $=2$, node score cut-off $=0.2, \mathrm{k}$-score $=2$ and max depth=100. Afterwards, the functional analysis of the most significant module was analyzed using Database for Annotation, Visualization and Integrated Discovery (DAVID) database version 6.8 (13)

GO functional annotation and KEGG pathway enrichment analyses. DAVID, an online bioinformatics database, was used to integrate biological data and functional annotation information of genes and proteins. The functions of DEGs were analyzed using the DAVID database. $\mathrm{P}<0.05$ was considered statistically significant. The GO database was used to annotate genes and identify the associated biological characteristics of the genes (14). KEGG, a database resource for functions of biological systems generated using high-throughput techniques, was conducted using Cytoscape software version 3.7.1 (15).

Identification and analysis of hub genes. Hub genes were identified using cytoHubba tool kits of Cytoscape (16). Top hub genes with $\geq 10$ degrees in $\mathrm{BC}$ samples were selected. Hub genes were identified and analyzed using the cBioPortal platform (17). The Biological Networks Gene Oncology (BiNGO) plug-in of Cytoscape was used to analyze the biological process of the hub genes (18). The University of California, Santa Cruz (UCSC) Cancer Genomics Browser was used to construct the clustering of hub genes in the TCGA database (19). Moreover, the Kaplan-Meier method was used to compare overall survival curves between different expression gene groups in the survival probability study. $\mathrm{P}<0.05$ was considered to indicate a statistically significant difference. Oncomine online database analyzed the expression of KIF11 between cancer and normal tissue in four different datasets (20-23). Meanwhile, the relationships between gene expressions and tumor grades were analyzed using the Oncomine online database.

Patients and BC samples. The present study was approved by The Research Ethics Committee of the Sun Yat-sen University Cancer Center (Guangzhou, China; approval no. B2020-198). The inclusion criteria were as follows: i) No history of any other malignant tumors; and ii) no anticancer therapy before surgery. The exclusion criteria were as follows: i) Other malignant tumors; and ii) preoperative treatments, such as adjuvant chemotherapy or radiation therapy. In total, 31 paired $\mathrm{BC}$ and adjacent tissues from 31 patients were obtained from the Sun Yat-sen University Cancer Center. The tissue samples were acquired by resection. The tissue samples were stored at $-80^{\circ} \mathrm{C}$ before fixation. Tissue were embedded in paraffin for long-term preservation. Written informed consent was obtained from enrolled patients. The BC tissues were confirmed by pathological diagnosis by independent pathologists. Clinicopathological data of patients with $\mathrm{BC}$ were also obtained, including age, sex, tumor stage, tumor grade, lymph node metastasis status, distant metastasis status and invasiveness.

Cell culture. Overall, four human bladder cell lines (Tcc-Sup, UM-UC-3, RT4 and J82) and a normal urinary tract cell line (HCV29) were cultured in RPMI-1640 (Invitrogen; Thermo Fisher Scientific, Inc.). Cells were supplemented with $10 \%$ fetal 
Table II. A total of 141 DEGs were identified from three profile datasets, including 55 upregulated and 86 downregulated genes in bladder cancer compared with normal tissues.

\section{DEGs}

Upregulated

Downregulated
Genes name
TPX2, CDK1, SAPCD2, AURKA, AURKB, PAFAH1B3, CELSR3, TYMS, MCM5, MELK, CCNA2, CDCA5, CKS2, CENPE, TACC3, CCNB2, PRC1, CDT1, CEP55, CDC45, NT5DC2, MCM2, TMEM74B, DLGAP5, RAD51AP1, CKAP2L, KIAA0101, SHMT2, KIF11, ASF1B, KIF2C, CENPA, CDC20, CENPN, BUB1, POLQ, TRIP13, TROAP, TK1, ASPM, SLC7A5, TOP2A, SLC16A3, CA2, HJURP, HMMR, KIF20A, TTK, NCAPG, SLC52A2, CENPM, FAM64A, CENPF, NUSAP1, CDCA8 CPXM2, MSRB3, LIMS2, CNN1, PALLD, HLA-DRA, CLEC3B, STON1, NDNF, FHL1, SORBS2, PLSCR4, CSRP1, ANKDD1A, PLAC9, LGALS4, RNF150, LAMC3, PDK4, REEP1, EFEMP1, BIN1, AXL, TCEAL2, RASL12, IGFBP6, P3H2, PID1, P2RX1, SRPX, ACACB, PGM5, PRAC1, GSTM5, SOBP, SH3GL2, PLPPR4, CYBRD1, SYNM, C2orf40, CFD, RNASE4, WLS, HSPB6, FAM129A, DACT3, CFH, FLNC, ANTXR2, ABCA8, LMOD1, ZBTB16, KCNMB1, DES, MYH11, KBTBD11, CPED1, PRICKLE2, FERMT2, DPT, MFAP4, MGLL, SCARA5, SDPR, SORBS1, CYGB, KLHL13, FGF9, TSHZ3, SPARCL1, ITM2A, COX7A1, ACTG2, TMOD1, TGFBR2, PCP4, FAM107A, GPX3, PTGS1, CASQ2, LPAR1, MAMDC2, ACOX2, EVA1C, NDN, FBLN5

DEGs, differentially expressed genes.

bovine serum (HyClone; Cyvita) and incubated at $37^{\circ} \mathrm{C}$ in a humidified atmosphere of $5 \% \mathrm{CO}_{2}$.

Reverse transcription-quantitative PCR $(R T-q) P C R$ analysis. Total RNA was isolated from tissues using TRIzol ${ }^{\circledR}$ reagent (Invitrogen; Thermo Fisher Scientific, Inc.) following the manufacturer's protocol. cDNA was generated by reverse transcription using a SuperScript III First-Strand cDNA system (Invitrogen; Thermo Fisher Scientific, Inc.) under the following conditions: $42^{\circ} \mathrm{C}$ for $50 \mathrm{~min}$ followed by heat inactivation for $5 \mathrm{~min}$ at $80^{\circ} \mathrm{C}$. The KIF11 sense primer was 5'-TAT TGAATGGGCGCTAGCTT-3', and the antisense primer was 5'-TCGTCTGCGAAGAAGAAAGA-3'. For the housekeeping gene GAPDH, the sense primer was 5'-ACCACAGTCCAT GCCATCAC-3' and the antisense primer was 5'-TCCACC ACCCTGTTGCTGTA-3'. RT-qPCR was performed using a 7900HT Fast Real-time PCR system (Thermo Fisher Scientific, Inc.) as follows: $50^{\circ} \mathrm{C}$ for $2 \mathrm{~min}, 95^{\circ} \mathrm{C}$ for $10 \mathrm{~min}, 40$ cycles of $95^{\circ} \mathrm{C}$ for $15 \mathrm{sec}$ and $60^{\circ} \mathrm{C}$ for $1 \mathrm{~min}$. The crossing threshold $(\mathrm{Cq})$ value was calculated and recorded using the instrument's software (SDS version 2.3). The mRNA expression data was normalized to GAPDH and calculated using the comparative threshold cycle $\left(2^{-\Delta \Delta \mathrm{Cq}}\right)$ method (24).

Immunohistochemistry (IHC). The tissue sample were immersed in $10 \%$ neutral buffered formalin for 3 days at room temperature $\left(27^{\circ} \mathrm{C}\right)$ for fixation. Samples were cut into $4-\mu \mathrm{m}$ thick paraffin sections. Paraffin sections were incubated in an oven at $60^{\circ} \mathrm{C}$ for $2 \mathrm{~h}$. Slides were boiled in citrate buffer ( $\mathrm{pH} \mathrm{6.0)}$ and immersed in $3 \%$ hydrogen peroxide. Slides were blocked in 5\% goat blocking serum (Thermo Fisher Scientific, Inc.) at room temperature for $1 \mathrm{~h}$. Next, the slides were incubated with anti-KIF11 antibody (1:500; cat. no. ab61199; Abcam) overnight at $4^{\circ} \mathrm{C}$. After rinsing thrice with phosphate-buffered saline $(0.01 \mathrm{~mol} / \mathrm{l}, \mathrm{pH} 7.4)$, the stained sections were incubated with goat anti-rabbit poly-horseradish peroxidase-conjugated secondary antibody (1:200; cat. no. 32260; Thermo Fisher Scientific, Inc.) for $30 \mathrm{~min}$ at room temperature and stained with 3,3-diaminobenzidine for $3 \mathrm{~min}$ at room temperature. Then, the tissue sections were counterstained with hematoxylin for $1 \mathrm{~min}$ at room temperature and dehydrated with graded ethanol $(75,85,95$ and $100 \%)$. These slides were observed using a light microscope (magnification, x100 and $\mathrm{x} 200$ ). Two experienced pathologists, who are independent from the present study group, calculated the H-Score of IHC staining based on the percentage score and intensity score of positively stained cells. The percentage of positively stained cells was scored as follows: $0=$ staining $0-5 \% ; 1=$ staining $6-25 \% ; 2=$ staining $26-50 \% ; 3=$ staining $51-75 \% ; 4=$ staining $>76 \%$. The intensity of positively stained cells was scored as follows: $0=$ absent staining; 1 =weak; $2=$ moderate; 3 =strong). The IHC score was calculated by multiplying the percentage and intensity score and ranged from 0 to 12 .

Western blotting. Total protein samples were extracted in lysis buffer (Vazyme Biotech, Co., Ltd.). Protein concentration was determined using a bicinchoninic acid assay kit. Protein samples (20 $\mu \mathrm{g}$ per lane) were separated using $12 \%$ SDS-PAGE. Electrophoresis was carried out at a constant voltage. Proteins were transferred onto polyvinylidene fluoride (PVDF) membranes (EMD Millipore). After electrophoresis, the PVDF membrane was sealed with $5 \%$ skimmed milk at room temperature for $60 \mathrm{~min}$. The membrane was incubated with rabbit anti-KIF11 antibody (1:500; cat. no. ab61199; Abcam) or rabbit anti-GAPDH antibody (1:2,000; cat. no. ab9485; Abcam) at $4^{\circ} \mathrm{C}$ overnight. After three 10 -min washes with phosphate-buffered saline-Tween (PBST, 0.05\% Tween-20), anti-rabbit horseradish peroxidase (HRP)-conjugated secondary antibody (1:5,000; cat. no. 7074; Cell Signaling Technology, Inc.) was added and incubated with the membrane for $1 \mathrm{~h}$ at room temperature. Secondary antibody was washed away with 

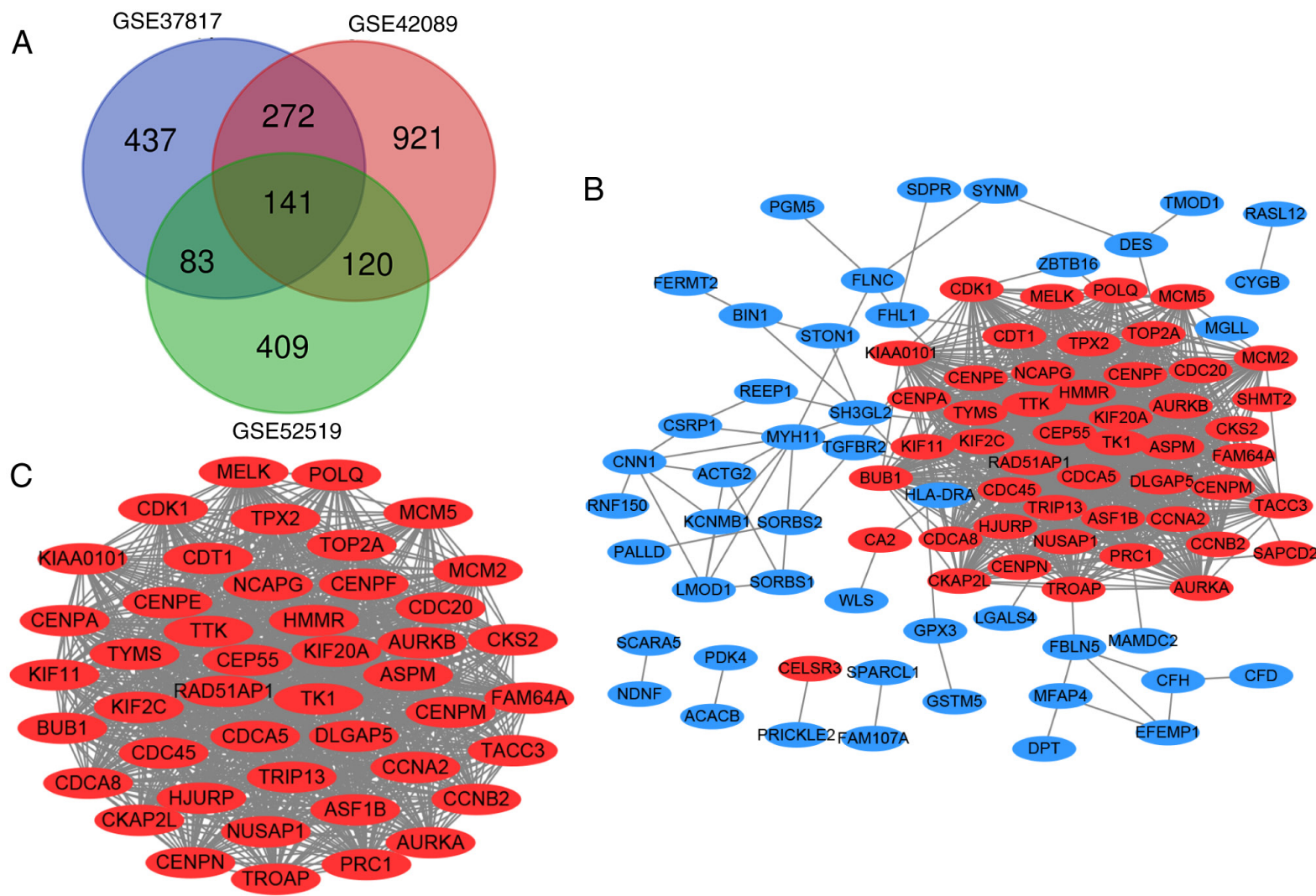

Figure 1. Venn diagram, PPI network and significant module of DEGs. (A) DEGs were selected among the mRNA expression profiling datasets GSE37817, GSE42089 and GSE52519. These three datasets showed an overlap of 141 genes in the Venn diagram. (B) PPI network of DEGs was constructed using Cytoscape. Red nodes represent upregulated genes, and blue nodes represent downregulated genes. (C) Most significant module was obtained from the PPI network with 45 nodes and 954 edges and exhibited the highest score using the Molecular Complex Detection plug-in. PPI, protein-protein interaction network. DEGs, differentially expressed genes.

Table III. GO and Kyoto Encyclopedia of Genes and Genomes pathway enrichment analyses of DEGs in the most significant module.

\begin{tabular}{llcr}
\hline Pathway ID & \multicolumn{1}{c}{ Pathway description } & Count in gene set & FDR \\
\hline GO:1903047 & Mitotic cell cycle process & 35 & $1.73 \times 10^{-32}$ \\
GO:0007049 & Cell cycle & 38 & $4.27 \times 10^{-28}$ \\
GO:0007067 & Mitotic nuclear division & 25 & $3.48 \times 10^{-24}$ \\
GO:0051301 & Cell division & 26 & $9.13 \times 10^{-23}$ \\
GO:0005819 & Spindle & 17 & $1.33 \times 10^{-13}$ \\
GO:0015630 & Microtubule cytoskeleton & 24 & $3.51 \times 10^{-12}$ \\
GO:0000793 & Condensed chromosome & 13 & $7.37 \times 10^{-10}$ \\
GO:0005524 & ATP binding & 17 & $4.35 \times 10^{-5}$ \\
GO:0032559 & Adenyl ribonucleotide binding & 17 & $6.09 \times 10^{-5}$ \\
GO:0030554 & Adenyl nucleotide binding & 17 & $6.74 \times 10^{-5}$ \\
hsa04110 & Cell cycle & 9 & $4.08 \times 10^{-8}$ \\
hsa04114 & Oocyte meiosis & 5 & 0.061 \\
\hline
\end{tabular}

GO, Gene Ontology; hsa, homo sapiens; FDR, false discovery rate.

PBST and the membranes were prepared for exposure. The PVDF membrane were detected using the hypersensitive ECL chemiluminescence kit (Beyotime Institute of Biotechnology). Two exposure reagents were mixed in equal proportion and applied to the PVDF membrane. The intensity was measured using Quantity One software (version 4.6.6; Bio-Rad Laboratories).

Statistical analysis. All the experiments were repeated three times. All of the statistical analyses were performed using 
A

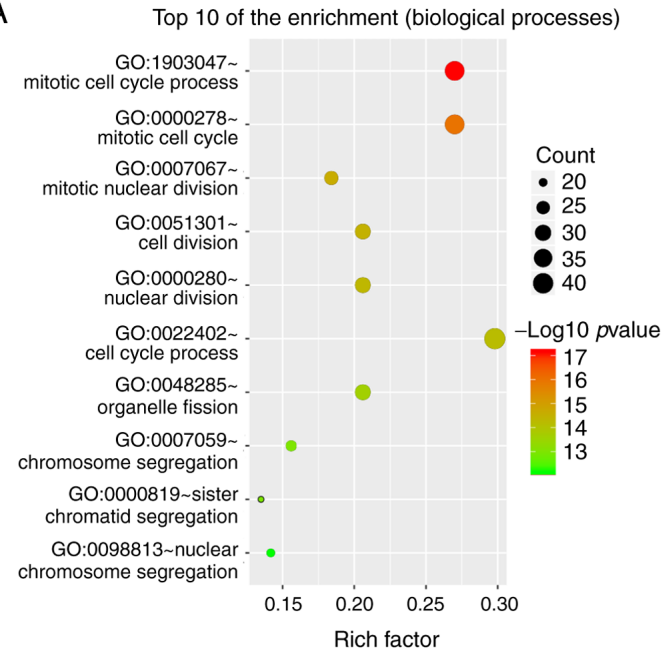

C Top 10 of the enrichment (molecular functions)

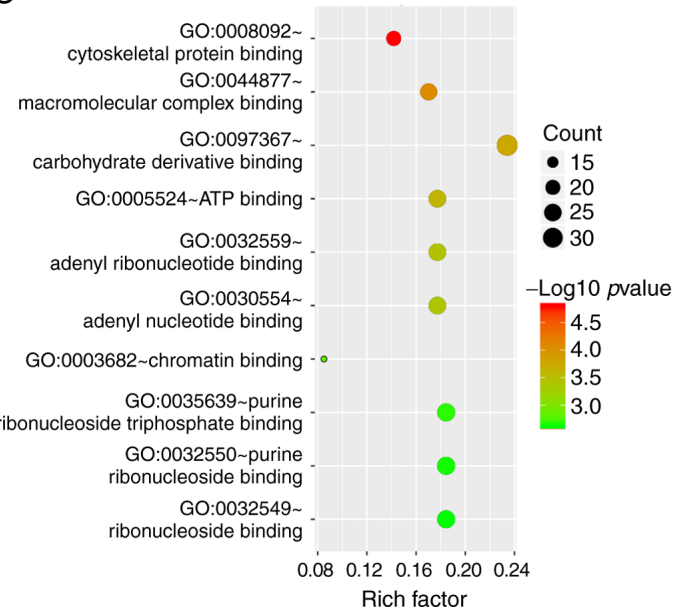

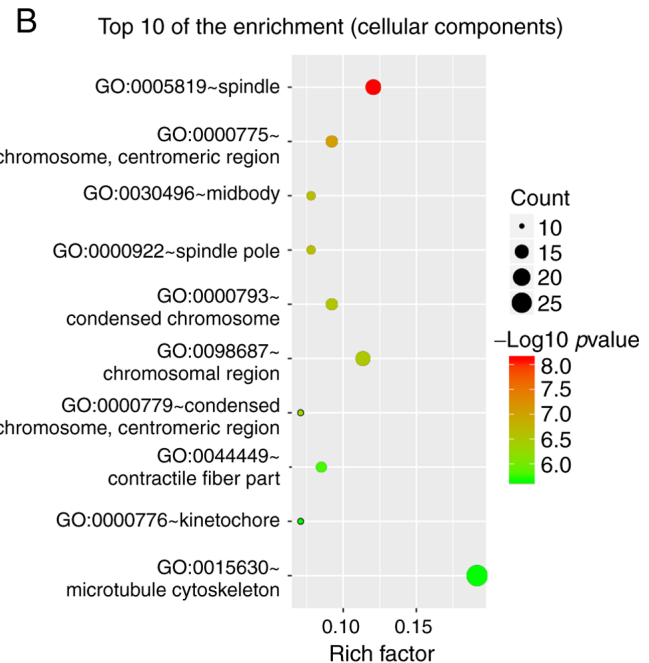

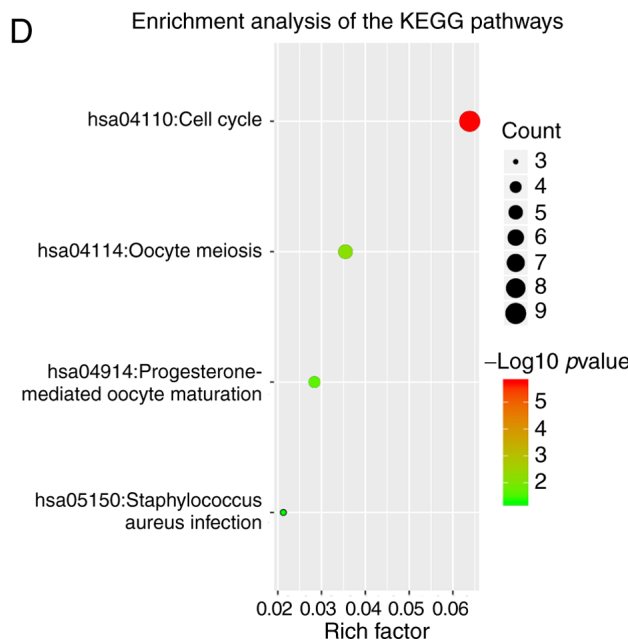

Figure 2. Gene function analysis of DEGs. GO functional enrichment of DEGs in (A) biological processes, (B) cellular component and (C) molecular function ontology. (D) KEGG pathway enrichment of DEGs. DEGs, the differentially expressed genes; GO, Gene Ontology; KEGG, Kyoto Encyclopedia of Genes and Genomes.

SPSS 25.0 (IBM Corp) and GraphPad Prism 8.0.1 (GraphPad Software). The data was analyzed using ANOVA followed by Dunnett's test, paired Student's t-tests (two-sided) or Fisher's exact tests (two-sided). Data was expressed as the means \pm standard deviation. $\mathrm{P}<0.05$ was considered to indicate a statistically significant difference.

\section{Results}

Identification of DEGs in BC. The GEO2R tool was used to identify the DEGs (933 in GSE37817, 1,454 in GSE42089 and 753 in GSE52519) in BC. A total of 141 overlapping DEGs were identified among the three datasets (Fig. 1A), consisting of 55 upregulated genes and 86 downregulated genes between $\mathrm{BC}$ and non-cancerous tissues. These DEGs were visualized using Venn diagrams and are listed in Table II.

PPI network construction and module analyses. A PPI network of the 141 DEGs was constructed to elucidate and investigate the PPIs (Fig. 1B). The MCODE plug-in of Cytoscape was used to identify the clusters in the PPI networks. (Fig. 1C). The cluster network consisted of 45 nodes and 954 edges.
Sequentially it was showed as follows: CKS2, ASPM, MELK, BUB1, DLGAP5, AURKB, CKAP2L, CCNB2, TK1, MCM2, CDC45, CEP55, HJURP, KIF20A, CDK1, KIAA0101, ASF1B, KIF11, HMMR, TPX2, CCNA2, CDCA5, AURKA, CENPM, NCAPG, CENPE, KIF2C, TACC3, CENPF, FAM64A, CENPN, RAD51AP1, TYMS, POLQ, CDT1, NUSAP1, MCM5, CDCA8, TROAP, PRC1, CDC20, TRIP13, TOP2A, TTK and CENPA. These node genes with high hub degrees may play critical roles in BC. Subsequently, the results indicated that the genes in the most significant module were mainly enriched in 'mitotic cell cycle process', 'cell cycle', 'mitotic nuclear division', 'cell division', 'adenyl nucleotide binding', 'ATP binding' and 'oocyte meiosis' (Table III).

GO functional annotation and KEGG pathway enrichment analysis. GO functional annotation and KEGG pathway enrichment analysis were used to investigate the biological functions of DEGs using DAVID. GO enrichment analysis results indicated that the DEGs in biological processes (BP) were mainly enriched in 'mitotic cell cycle process', 'organelle fission', 'nuclear/cell division' and 'chromosome segregation’ (Fig. 2A). Moreover, the DEGs in cell component were 
A
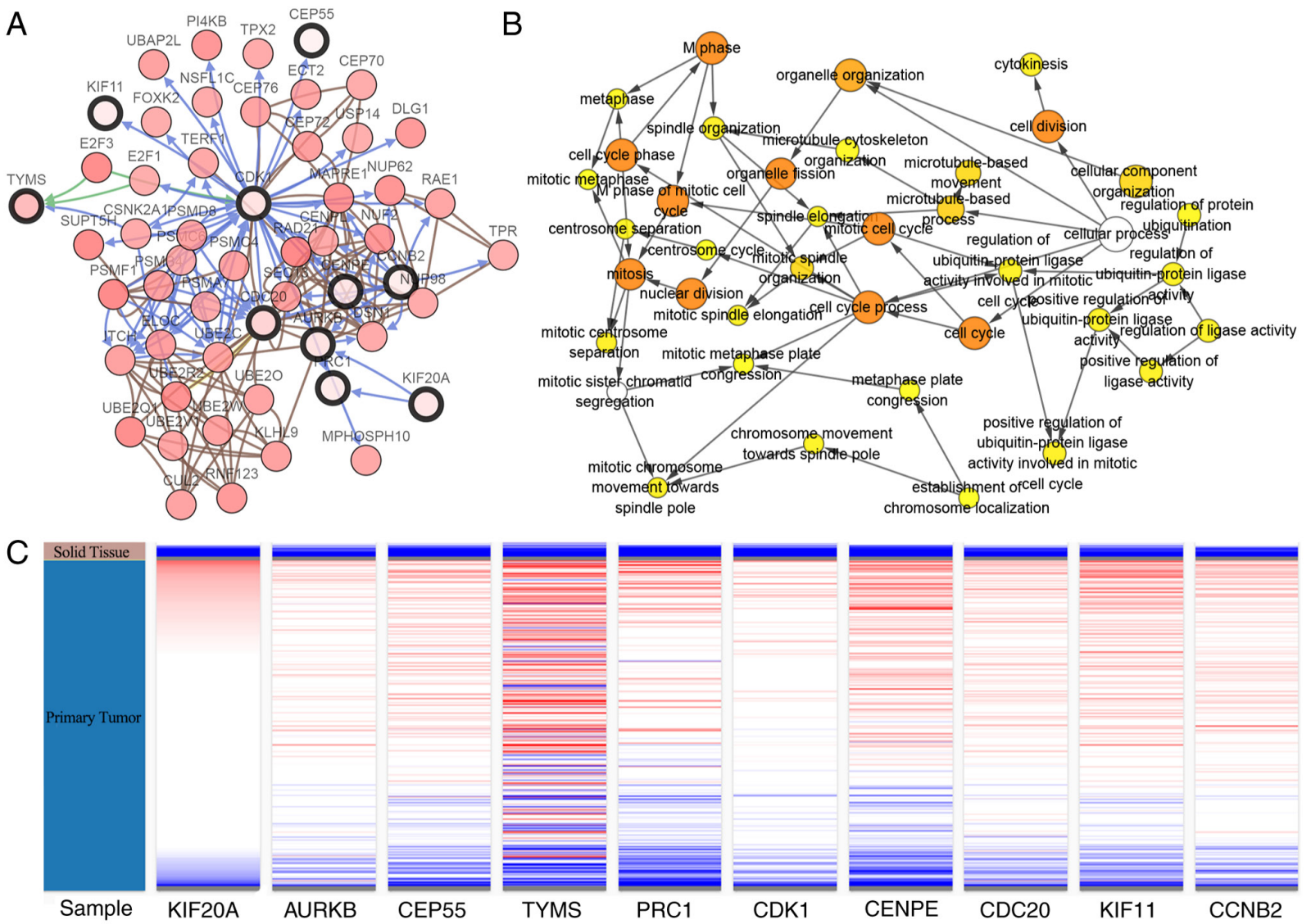

Figure 3. Interaction network, biological process analysis and hierarchical clustering of the hub genes. (A) Hub genes and their co-expression genes were visualized using the cBioPortal. Nodes with bold black outlines represent hub genes. Nodes with thin black outlines represent co-expression genes. (B) Biological process analysis of hub genes was constructed using the Biological Networks Gene Oncology plug-in. Color depth of the nodes refers to the corrected $\mathrm{P}$-value of ontologies. Size of the nodes refers to the numbers of genes that are involved in the ontologies. $\mathrm{P}<0.05$ was considered statistically significant. (C) Hierarchical clustering of hub genes was constructed using the University of California, Santa Cruz online database. Solid tissue samples above the blue bar are non-cancerous samples and the samples under the brown bar are tumor samples. Upregulation of genes is marked in red; downregulation of genes is marked in blue.

enriched in 'spindle', 'condensed chromosome', 'midbody' and 'microtubule cytoskeleton' (Fig. 2B). Alterations in molecular function (MF) were abundant in 'carbohydrate derivative binding', 'cytoskeletal protein binding', 'ATP binding', 'ribonucleoside binding' and 'chromatin binding' (Fig. 2C). The KEGG pathway enrichment analysis revealed that the DEGs were mainly abundant in 'cell cycle', 'oocyte meiosis' and 'progesterone-mediated oocyte maturation' (Fig. 2D).

Identification and analysis of hub genes. According to the degree score generated by the cytohubba plug-in, the top ten genes, including TYMS, AURKB, CDK1, CCNB2, CEP55, KIF20A, KIF11, CENPE, PRC1 and CDC20, were identified as potential hub genes. A co-expression network of these genes was generated for analysis using the cBioPortal platform (Fig. 3A). Moreover, the BiNGO plug-in analyzed the biological process analyses of the hub genes. The alterations were enriched in cell cycle process, mitosis, mitotic spindle organization, organelle organization, regulation of ubiquitin-protein ligase activity and establishment of chromosome localization (Fig. 3B). Subsequently, the UCSC Cancer Genomics Browser was used to analyze hierarchical clustering. Hierarchical clustering showed that the hub genes could differentiate bladder tumor tissues from non-cancerous tissues (Fig. 3C). These results may provide insight into how cancerous tissues differentiate from normal bladder tissues.

KIF11 is a promising gene target in bladder cancer. Among the identified hub genes, KIF11 was connected to 36 nodes in the cluster network constructed using Cytoscape, indicating it may play significant roles in the progression or carcinogenesis of BC. The overall survival of KIF11 was performed using Kaplan-Meier curve according to the data from cBioPortal. Patients with BC with KIF11 mRNA alterations exhibited a poorer overall survival rate $(\mathrm{P}=0.011$; Fig. $4 \mathrm{~A})$. As for the patient samples included in IHC analysis, the overall survival rate of patients with high KIF11 expression was lower compared with that in patients with low KIF11 expression ( $\mathrm{P}=0.030$; Fig. 4B). Moreover, Oncomine analysis of cancer vs. normal tissue indicated that KIF11 was overexpressed in BC in four different datasets, including Dyrskjot Bladder 3 (20), Lee Bladder (21), Sanchez-Carbayo Bladder 2 (22) and Sanchez-Carbayo Bladder 2 (23) ( $\mathrm{P}<0.001$; Fig. 4C). In the Sanchez-Carbayo Bladder 2 dataset, three groups in different tumor grade were compared, and higher expression levels of KIF11 mRNA were associated with tumor grade (22) (P<0.001; Fig. 4D). 


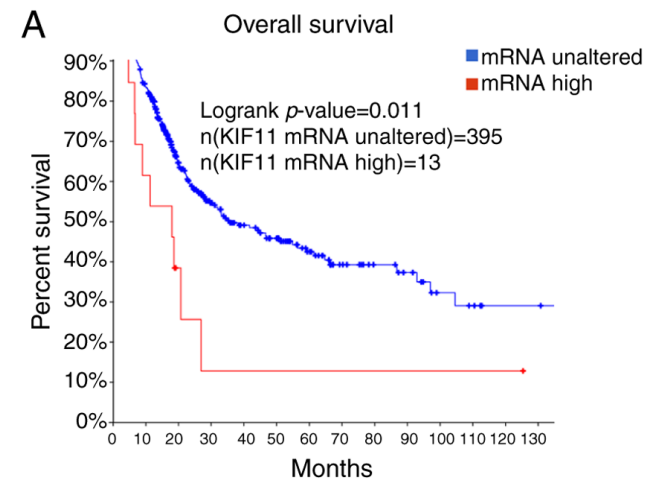

B Overall survival of the patients included in $\mathrm{IHC}$ analysis

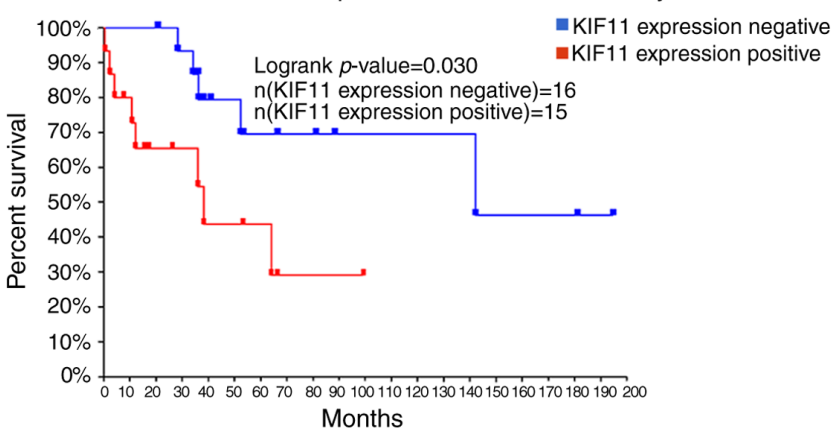

C Comparison of KIF11 across 4 analyses

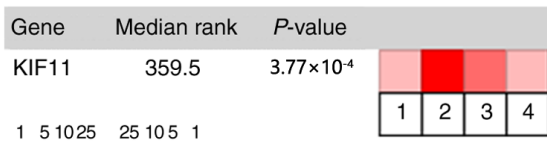

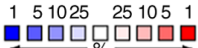

1. $P$-value $=6.94 \times 10^{-5}$ fold-change $=1.748$

2. $P$-value $=9.22 \times 10^{-8}$ fold-change $=1.870$

3. $P$-value $=2.32 \times 10^{-14}$ fold -change $=4.024$

4. $P$-value $=7.54 \times 10^{-4}$ fold-change $=2.251$

D KIF11 expression in Sanchez-Carbayo bladder 2

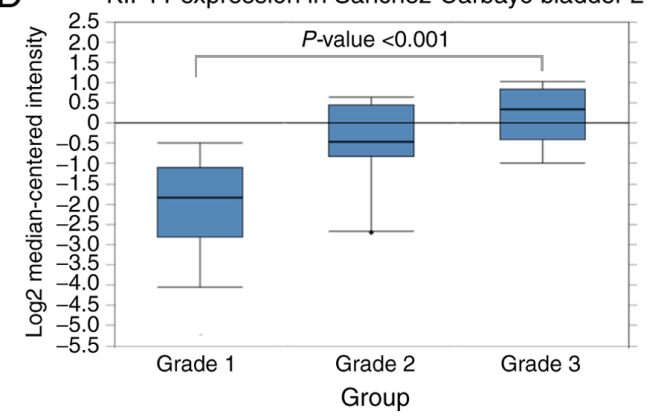

Figure 4. Overall survival, Oncomine analysis and association between KIF11 mRNA expression and tumor grade. (A) Overall survival analysis of hub gene was performed using the Kaplan-Meier analysis. (B) Overall survival analysis of the patients included in immunohistochemistry analysis was performed using the Kaplan-Meier curve. (C) Oncomine online database analysis of cancer vs. normal tissue of KIF11. Heat maps of KIF11 gene expression in clinical bladder cancer samples vs. normal tissues. 1. Infiltrating Bladder Urothelial Carcinoma vs. Normal Dyrskjot Bladder 3 (20). 2. Infiltrating Bladder Urothelial Carcinoma vs. Normal Lee Bladder (21). 3. Infiltrating Bladder Urothelial Carcinoma vs. Normal Sanchez-Carbayo Bladder 2 (22). 4. Bladder Urothelial Carcinoma Type: Infiltrating Bladder Urothelial Carcinoma Stransky Bladder (23). (D) Association between KIF11 mRNA expression levels and tumor grade in BC samples in the Sanchez-Carbayo Bladder 2 dataset. KIF11, kinesin family member 11.
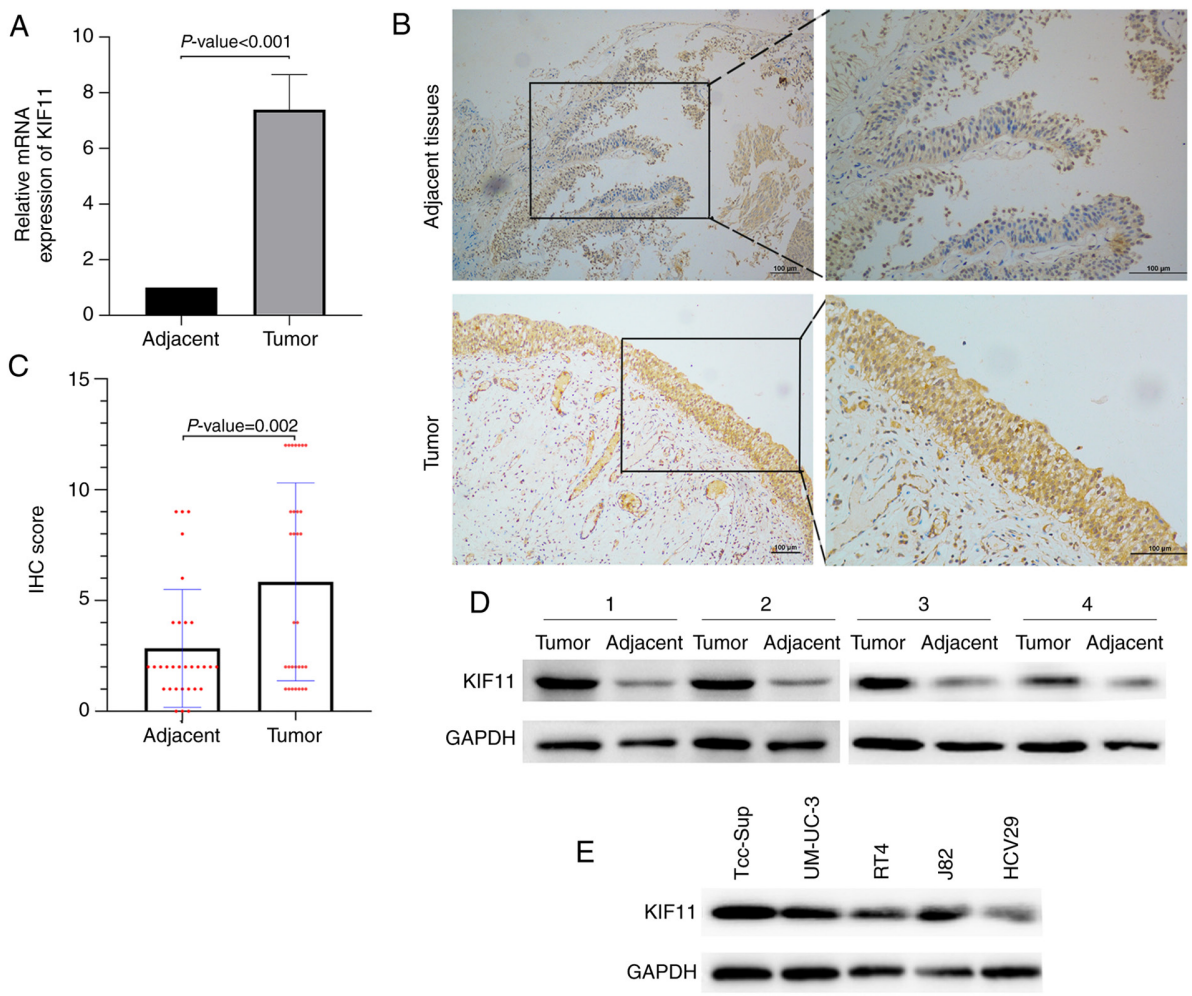

Figure 5. Expression of KIF11 mRNA and protein in BC and adjacent tissue samples. (A) Expression of KIF11 mRNA in BC and adjacent tissue samples. (B) IHC analysis of KIF11 protein expression in BC and adjacent tissues. (C) IHC score of KIF11 protein expression in BC and adjacent tissues. (D) Western blot analysis of KIF11 protein expression in four pairs of BC and adjacent tissues. (E) Western blot analysis of KIF11 protein expression in human BC cell lines Tcc-Sup, UM-UC-3, RT4 and J82 and the normal urinary tract cell lines (HCV29). KIF11, kinesin family member 11; BC, bladder cancer; IHC, immunohistochemistry. 
Table IV. Association between KIF11 expression and clinicopathological characteristics of bladder cancer patients included in IHC analysis.

\begin{tabular}{|c|c|c|c|c|}
\hline \multirow[b]{2}{*}{ Variables } & \multirow[b]{2}{*}{ Patients } & \multicolumn{2}{|c|}{ KIF11 expression } & \multirow[b]{2}{*}{ P-value } \\
\hline & & Positive, $\mathrm{n}=15$ & Negative, $n=16$ & \\
\hline Age, years & & & & 0.473 \\
\hline$<65$ & 18 & 10 & 8 & \\
\hline$\geq 65$ & 13 & 5 & 8 & \\
\hline Sex & & & & 0.433 \\
\hline Male & 22 & 12 & 10 & \\
\hline Female & 9 & 3 & 6 & \\
\hline Tumor stage & & & & 0.035 \\
\hline Ta/Tis/T1 & 11 & 2 & 9 & \\
\hline $\mathrm{T} 2$ & 13 & 9 & 4 & \\
\hline $\mathrm{T} 3 / \mathrm{T} 4$ & 7 & 4 & 3 & \\
\hline Tumor grade & & & & 0.046 \\
\hline G1 & 8 & 1 & 7 & \\
\hline $\mathrm{G} 2$ & 5 & 2 & 3 & \\
\hline G3 & 18 & 12 & 6 & \\
\hline Lymph node metastasis & & & & 1.000 \\
\hline Negative & 25 & 12 & 13 & \\
\hline Positive & 6 & 3 & 3 & \\
\hline Distant metastasis & & & & 0.654 \\
\hline No & 26 & 12 & 14 & \\
\hline Yes & 5 & 3 & 2 & \\
\hline Invasiveness & & & & 0.023 \\
\hline Non-invasive & 11 & 2 & 9 & \\
\hline Invasive & 20 & 13 & 7 & \\
\hline
\end{tabular}

${ }^{\text {a}}$ Fisher's exact test.

KIF11 expression is upregulated in BC samples and bladder cells. The mRNA expression level of KIF11 was examined in $31 \mathrm{BC}$ and adjacent tissues. RT-qPCR analysis revealed that KIF11 was highly expressed in BC tissues compared with the adjacent tissues $(\mathrm{P}<0.001$; Fig. 5A). Subsequently, protein expression levels of KIF11 were analyzed using IHC (Fig. 5B). Increased IHC score of KIF11 was observed in BC samples compared with the adjacent tissues ( $\mathrm{P}=0.002$; Fig. $5 \mathrm{C})$. The clinicopathological characteristics of patients with $\mathrm{BC}$ were included in the IHC analysis and their associations with KIF11 expression are presented in Table IV. The age at diagnosis ranged from 26 to 85 years and there were 22 males and 9 females. The results suggested that KIF11 expression was significantly associated with tumor stage $(\mathrm{P}=0.035)$, tumor grade $(\mathrm{P}=0.046)$ and invasiveness $(\mathrm{P}=0.023)$. However, KIF11 expression was not associated with age, sex, lymph node metastasis and distant metastasis. Furthermore, western blot analysis showed that the expression of the KIF11 protein was higher in these four samples compared with in the adjacent normal tissues (Fig. 5D). Also, the expression of the KIF11 protein was upregulated in four human bladder cell lines (Tcc-Sup, UM-UC-3, RT4 and J82) compared with a normal urinary tract cell line (HCV29) (Fig. 5E). Together, these results indicated increased expression of KIF11 mRNA and protein in bladder cancer at both the transcriptional and translational levels.

\section{Discussion}

Bioinformatics plays a significant role in current tumor research, contributing to a better understanding of carcinogenesis using systematic bioinformatic methods (25). Novel biomarkers with high prognostic efficiency are needed. Microarray technology has become a method for exploring genetic alterations in $\mathrm{BC}$ and identifying potentially useful biomarkers (26).

KIF11 belongs to the kinesin- 5 family of proteins and has functions in numerous physiological functions, including cell cycle, cell mitosis and intracellular vesicle transport(27). Several studies have shown that KIF11 is upregulated in a wide variety of tumors, such as gastric, oral and breast cancer, meningioma, clear cell renal cell carcinoma and prostate cancer (28-33). However, the interaction among BC and KIF11 has not been widely reported and the levels of expression and biological functions of KIF11 in BC remain unclear. In the present study, 
three microarray datasets were used to identify potential candidate genes in BC. A total of 141 DEGs was identified, including 55 upregulated genes and 86 downregulated genes. The top ten DEGs caused significant alterations in GO enrichment and KEGG pathway analysis. GO enrichment suggested that the DEGs were associated with the cell cycle, cell division, mitotic nuclear division, chromosome segregation, sister chromatid cohesion and mitotic cytokinesis. Moreover, the KEGG pathway enrichment analysis showed associations with cell cycle, oocyte meiosis and progesterone-mediated oocyte maturation. Cell division and cell cycle are the basic processes in cell proliferation, and their abnormalities contribute to carcinogenesis and tumor progression (34). Ten hub genes, including TYMS, AURKB, CDK1, CCNB2, CEP55, KIF20A, KIF11, CENPE, PRC1 and CDC20 were identified according to PPI network of DEGs analyses. In previous studies, worse survival outcomes in bladder cancer patients were observed for high AURKB levels (35). High co-expression of TFCP2L1 and CDK1 in tumor tissues of patients with bladder cancer is associated with unfavorable clinical features, including high tumor grade and distant metastasis (36). CCNB2 is overexpressed in bladder cancer, and the downregulation of CCNB2 expression in bladder cancer inhibits cell invasion and migration (37). These previous studies clarified the importance of hub genes in BC. In the present study, KIF11 was connected to 36 nodes in the cluster network and was the most significant gene, indicating it may play significant roles in the progression or carcinogenesis of BC.

The overall survival analysis indicated that patients with BC with alterations in KIF11 mRNA or with elevated KIF11 expression exhibited poorer overall survival rate. To verify this finding, the ONCOMINE database was used to evaluate the mRNA level of KIF11 in other datasets and it revealed that KIF11 overexpression was common in BC in four different datasets. Furthermore, the upregulation of KIF11 was associated with tumor grade. Also, Daigo et al (29) observed that the overexpression of KIF11 in oral cancer is associated with advanced pathological (p) Tumor stage and advanced pNode stage, which is consistent with the present results that KIF11-overexpression was significantly associated with tumor stage (29). Moreover, 31 paired BC and adjacent tissues were analyzed and upregulation of KIF11 in BC samples and bladder cells was further validated using RT-qPCR, IHC analysis and western blotting. The current findings suggested that KIF11 could be used as a potential prognostic biomarker in BC.

In summary, the present study aimed to identify DEGs that may be involved in progression or carcinogenesis of $\mathrm{BC}$ and KIF11 was identified as a potential useful candidate gene. However, further studies with larger sample sizes should be performed to validate the present findings. Also, further investigation is required to elucidate the molecular mechanisms of KIF11. Soft agar colony formation and Transwell assays should be used to investigate the effect of the KIF11 proteins on the clonability and invasiveness of the BC cells. As KIF11 is overexpressed in tumor cells and tissues, it is speculated that the change may also exist in circulating tumor cells.

\section{Acknowledgements}

Not applicable.

\section{Funding}

The study was funded by The National Key Research and Development Program of China (grant no. Q2018YFC1313400) and The Natural Science Foundation of Guangdong Province (grant no. 2018A0303130344).

\section{Availability of data and materials}

The data used to support the findings of this study are available from the corresponding author upon request. The datasets analyzed during the current study are available in the GEO (https://www.ncbi.nlm.nih.gov/geo/), DAVID (http://david.ncifcrf.gov), STRING (http://string-db.org), GO (https://www.geneontology.org), KEGG (https://www.genome. jp/kegg/), Oncomine (http://www.oncomine.com) and TCGA (https://cancergenome.nih.gov/) databases.

\section{Authors' contributions}

XCM, ZTZ, DSW and JCX conceived of the study and performed the experiments. XCM, ZTZ, DSW, JCX, MJS, ZQZ, JXZ, YFD, FZS, JYY, JYH and YH collected data. XCM, DSW, JCX ZTZ and MJS contributed to the writing of the manuscript. MJS, ZQZ, JXZ, YFD, FZS, JYY, JYH and YH contributed to the data analysis. DSW and JCX supervised the study. DSW, JCX and MJS confirm the authenticity of all raw data. DSW and JCX revised the manuscript for important intellectual content. All the authors read and approved the final manuscript.

\section{Ethics approval and consent to participate}

The study was performed according to the principles of the Declaration of Helsinki and was approved by The Research Ethics Committee of Sun Yat-sen University (Guangzhou, China). Written informed consent was obtained from each patient.

\section{Patient consent for publication}

Not applicable.

\section{Competing interests}

The authors declare that they have no competing interests.

\section{References}

1. Siegel RL, Miller KD and Jemal A: Cancer statistics, 2019. CA Cancer J Clin 69: 7-34, 2019.

2. Cai Q, Chen Y, Xin S, Zhang D, Pan J, Xie Z, Xu C, Li S, Zhang X, Gao Y, et al: Temporal trends of bladder cancer incidence and mortality from 1990 to 2016 and projections to 2030. Transl Androl Urol 9: 153-165, 2020.

3. Zhou B, Zhang P, Wang Y, Shi S, Zhang K, Liao H and Zhang L: Interleukin-17 gene polymorphisms are associated with bladder cancer in a Chinese Han population. Mol Carcinog 52: 871-878, 2013.

4. Pençe S, Özbek E, Ozan Tiryakioğlu N and Ersoy Tunali N: Deregulation of seven $\mathrm{CpG}$ island-harboring miRNAs in bladder cancer: miR-155 and miR-23b as the most promising oncomiRs. Cell Mol Biol (Noisy-le-grand) 62: 25-30, 2016. 
5. Wang L, Smith BA, Balanis NG, Tsai BL, Nguyen K, Cheng MW, Obusan MB, Esedebe FN, Patel SJ, Zhang H, et al: A genetically defined disease model reveals that urothelial cells can initiate divergent bladder cancer phenotypes. Proc Natl Acad Sci USA 117: 563-572, 2020 .

6. Deng YB, Nagae G, Midorikawa Y, Yagi K, Tsutsumi S, Yamamoto S, Hasegawa K, Kokudo N, Aburatani H and Kaneda A: Identification of genes preferentially methylated in hepatitis $\mathrm{C}$ virus-related hepatocellular carcinoma. Cancer Sci 101: 1501-1510, 2010

7. Zhou N, Singh K, Mir MC, Parker Y, Lindner D, Dreicer R, Ecsedy JA, Zhang Z, Teh BT, Almasan A and Hansel DE: The investigational aurora kinase A inhibitor MLN8237 induces defects in cell viability and cell-cycle progression in malignant bladder cancer cells in vitro and in vivo. Clin Cancer Res 19 1717-1728, 2013.

8. Borisov N,Tkachev V, Suntsova M, Kovalchuk O,Zhavoronkov A, Muchnik I and Buzdin A: A method of gene expression data transfer from cell lines to cancer patients for machine-learning prediction of drug efficiency. Cell Cycle 17: 486-491, 2018.

9. Edgar R, Domrachev M and Lash AE: Gene expression omnibus: NCBI gene expression and hybridization array data repository. Nucleic Acids Res 30: 207-210, 2002

10. Franceschini A, Szklarczyk D, Frankild S, Kuhn M, Simonovic M, Roth A, Lin J, Minguez P, Bork P, von Mering C and Jensen LJ: STRING v9.1: Protein-protein interaction networks, with increased coverage and integration. Nucleic Acids Res 41 (Database Issue): D808-D815, 2013.

11. Smoot ME, Ono K, Ruscheinski J, Wang PL and Ideker T: Cytoscape 2.8: New features for data integration and network visualization. Bioinformatics 27: 431-432, 2011.

12. Bandettini WP, Kellman P, Mancini C, Booker OJ, Vasu S, Leung SW, Wilson JR, Shanbhag SM, Chen MY and Arai AE: MultiContrast delayed enhancement (MCODE) improves detection of subendocardial myocardial infarction by late gadolinium enhancement cardiovascular magnetic resonance: A clinical validation study. J Cardiovasc Magn Reson 14: 83 , 2012.

13. Huang DW, Sherman BT, Tan Q, Collins JR, Alvord WG, Roayaei J, Stephens R, Baseler MW, Lane HC and Lempicki RA: The DAVID gene functional classification tool: A novel biological module-centric algorithm to functionally analyze large gene lists. Genome Biol 8: R183, 2007.

14. Ashburner M, Ball CA, Blake JA, Botstein D, Butler $\mathrm{H}$, Cherry JM, Davis AP, Dolinski K, Dwight SS, Eppig JT, et al: Gene ontology: Tool for the unification of biology. The gene ontology consortium. Nat Genet 25: 25-29, 2000.

15. Kanehisa M: The KEGG database. Novartis Found Symp 247: 91-103, 119-128, 244-152, 2002.

16. Chin CH, Chen SH, Wu HH, Ho CW, Ko MT and Lin CY: cytoHubba: Identifying hub objects and sub-networks from complex interactome. BMC Syst Biol 8 (Suppl 4): S11, 2014.

17. Gao J, Aksoy BA, Dogrusoz U, Dresdner G, Gross B, Sumer SO, Sun Y, Jacobsen A, Sinha R, Larsson E, et al: Integrative analysis of complex cancer genomics and clinical profiles using the cBioPortal. Sci Signal 6: pl1, 2013.

18. Maere S, Heymans K and Kuiper M: BiNGO: A cytoscape plugin to assess overrepresentation of gene ontology categories in biological networks. Bioinformatics 21: 3448-3449, 2005.

19. Kent WJ, Sugnet CW, Furey TS, Roskin KM, Pringle TH, Zahler AM and Haussler D: The human genome browser at UCSC. Genome Res 12: 996-1006, 2002.

20. Dyrskjøt L, Kruhøffer M, Thykjaer T, Marcussen N, Jensen JL, Møller K and Ørntoft TF: Gene expression in the urinary bladder: A common carcinoma in situ gene expression signature exists disregarding histopathological classification. Cancer Res 64 4040-4048, 2004.

21. Lee JS, Leem SH, Lee SY, Kim SC, Park ES, Kim SB, Kim SK, Kim YJ, Kim WJ and Chu IS: Expression signature of E2F1 and its associated genes predict superficial to invasive progression of bladder tumors. J Clin Oncol 28: 2660-2667, 2010.
22. Sanchez-Carbayo M, Socci ND, Lozano J, Saint F and Cordon-Cardo C: Defining molecular profiles of poor outcome in patients with invasive bladder cancer using oligonucleotide microarrays. J Clin Oncol 24: 778-789, 2006.

23. Stransky N, Vallot C, Reyal F, Bernard-Pierrot I, de Medina SG, Segraves R, de Rycke Y, Elvin P, Cassidy A, Spraggon C, et al: Regional copy number-independent deregulation of transcription in cancer. Nat Genet 38: 1386-1396, 2006.

24. Livak KJ and Schmittgen TD: Analysis of relative gene expression data using real-time quantitative PCR and the 2(-Delta Delta C(T)) method. Methods 25: 402-408. 2001.

25. Singer J, Irmisch A, Ruscheweyh HJ, Singer F, Toussaint NC, Levesque MP, Stekhoven DJ and Beerenwinkel N: Bioinformatics for precision oncology. Brief Bioinform 20: 778-788, 2019.

26. Li PC: Overview of microarray technology. Methods Mol Biol 1368: 3-4, 2016.

27. Whitehead CM and Rattner JB: Expanding the role of HsEg5 within the mitotic and post-mitotic phases of the cell cycle. J Cell Sci 111: 2551-2561, 1998

28. Imai T, Oue N, Nishioka M, Mukai S, Oshima T, Sakamoto N, Sentani K, Matsusaki K, Yoshida K and Yasui W: Overexpression of KIF11 in gastric cancer with intestinal mucin phenotype. Pathobiology 84: 16-24, 2017.

29. Daigo K, Takano A, Thang PM, Yoshitake Y, Shinohara M, Tohnai I, Murakami Y, Maegawa J and Daigo Y: Characterization of KIF11 as a novel prognostic biomarker and therapeutic target for oral cancer. Int J Oncol 52: 155-165, 2018.

30. Zhou J, Chen WR, Yang LC, Wang J, Sun JY, Zhang WW, He ZY and Wu SG: KIF11 functions as an oncogene and is associated with poor outcomes from breast cancer. Cancer Res Treat 51: 1207-1221, 2019.

31. Jungwirth G, Yu T, Moustafa M, Rapp C, Warta R, Jungk C, Sahm F, Dettling S, Zweckberger K, Lamszus K, et al: Identification of KIF11 as a novel target in meningioma. Cancers (Basel) 11: 545, 2019.

32. Jin Q, Dai Y, Wang Y, Zhang S and Liu G: High kinesin family member 11 expression predicts poor prognosis in patients with clear cell renal cell carcinoma. J Clin Pathol 72: 354-362, 2019.

33. Piao XM, Byun YJ, Jeong P, Ha YS, Yoo ES, Yun SJ and Kim WJ: Kinesin family member $11 \mathrm{mRNA}$ expression predicts prostate cancer aggressiveness. Clin Genitourin Cancer 15: 450-454, 2017.

34. Chen L, Yuan L, Qian K, Qian G, Zhu Y, Wu CL, Dan HC, Xiao Y and Wang X: Identification of biomarkers associated with pathological stage and prognosis of clear cell renal cell carcinoma by co-expression network analysis. Front Physiol 9: 399, 2018

35. Burgess EF, Livasy C, Trufan S, Hartman A, Guerreri R, Naso C, Clark PE, Grigg C, Symanowski J and Raghavan D: High aurora kinase expression identifies patients with muscle-invasive bladder cancer who have poor survival after neoadjuvant chemotherapy. Urol Oncol 37: 900-906, 2019.

36. Heo J, Noh BJ, Lee S, Lee HY, Kim Y, Lim J, Ju H, Yu HY, Ryu CM, Lee PC, et al: Phosphorylation of TFCP2L1 by CDK1 is required for stem cell pluripotency and bladder carcinogenesis. EMBO Mol Med 12: e10880, 2020.

37. Lei CY, Wang W, Zhu YT, Fang WY and Tan WL: The decrease of cyclin B2 expression inhibits invasion and metastasis of bladder cancer. Urol Oncol 34: 237.e1-e10, 2016.

This work is licensed under a Creative Commons Attribution-NonCommercial-NoDerivatives 4.0 International (CC BY-NC-ND 4.0) License. 